\title{
Design and Development of Lie Detector
}

\author{
Mr. Anjaneya L H \\ Associate Professor \\ Department of E\&EE \\ Bapuji Institute of Engineering and Technology, \\ Davangere, Karnataka, India - 577004 \\ Mrs. Shubha V Patel \\ Assistant Professor \\ Department of E\&IE \\ Bapuji Institute of Engineering and Technology, \\ Davangere, Karnataka, India - 577004
}

\author{
Ms. Deepa \\ Assistant Professor \\ Department of E\&CE \\ Bapuji Institute of Engineering and Technology, \\ Davangere, Karnataka, India - 577004 \\ Mr. Ali I K \\ Assistant Professor \\ Department of E\&CE \\ Bapuji Institute of Engineering and Technology, \\ Davangere, Karnataka, India - 577004
}

\begin{abstract}
Multi parameters like skin temperature, body resistance, heart rate, electrocardiogram, pressure of end tidal cardbondioxide, oxygen saturation are utilized to design a lie detector device. Skin temperature measure by temperature sensor monitors the difference in temperature during the procedure. Optical reflective sensor extracts the signal of heart rate. The changes in body resistance during the questionnaire is monitored using body resistance sensor. The acquired signals are transmitted to the computer via USB communication and processed.
\end{abstract}

Keywords-Skin temperature, body resistance, heart rate, heart rate variability, temperture sensor, polygraph.

\section{INTRODUCTION}

It has been assumed that lying is accompanied by a change in the body's physiological activity. The polygraph is a set of equipment that accurately measures various sorts of bodily activity such as heart rate, blood pressure, respiration, and palmar sweating. In recent years brain activity has also begun to be measured in this setting. This bodily activity can be displayed via ink writing pens on to charts or via a computer's visual display unit.

The polygraph is widely used by analytical staff in a variety of medical and scientific settings for purposes other than lie detection.

In lie detection situations, its use is based on the premise that lying is accompanied by changes in the activity measured by the polygraph. One of the major topics that psychologists and others have focused on across the decades is how best to determine if a testing procedure can be relied upon.

The overall objective of the work is to build a costeffective lie detector monitoring device and to construct a prototype system with a low cost. A high performance microcontroller is used to develop technologies for a personal aid for mobility and monitoring.

The principle is based on the human skin resistance, heart rate and body temperature that changes according to truthful response of subject to the questionnaire. If person lie, the skin conductance increases with increase of secretion from sweat glands thereby decreasing skin resistance. Other physiological parameters like the skin temperature and heart rate increases in response to lie by the subject. These physiological parameters are good indicators of subject's false and lie statement in the current work.

The work offers an alternative strategy to measure skin resistance, body temperature and heart rate, by designing and implementing a low cost, low powered, non-intrusive and accurate measurement that monitors the input parameters and displays the output.

\section{METHODOLOGY}

The approach of this work is to keep things as simple as possible. The system mainly carries out signal acquisition and pre-processing. It consists of two parts: Hardware and software. The hardware component consists of a power supply, sensor and amplifier. The software part consists of analysis of input signals from Arduino IDE.

The circuit will be operating on its USB portable power source. The power source and circuitry will provide adequate voltage for the electronic components to function properly. There will be a sensor to convert the input signal into electrical signal. The signal will be convert analog to digital from Arduino IDE.

\section{A. Hardware description}

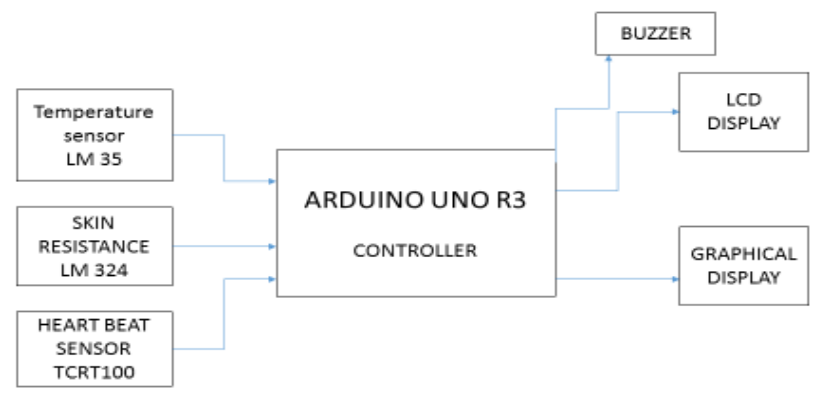

Fig 1. Block diagram of lie detector

The figure 1 shows the block diagram of lie detector.

The LM35 temperature sensor series are precision integrated-circuit temperature plastic TO-92 transistor package. The LM35D is also avail sensor, whose output voltage is linearly proportional to the able in an 8-lead 
surface mount small outline package and a Celsius temperature.

The LM324 series are low-cost, quad operational amplifiers with true differential inputs. They have several distinct advantages over standard operational amplifier types in single supply applications. The quad amplifier can operate at supply voltages as low as $3.0 \mathrm{~V}$ or as high as $32 \mathrm{~V}$ with quiescent currents about one-fifth of those associated with the MC1741. The common mode input range includes the negative supply, thereby eliminating the necessity for external biasing components in many applications. The output voltage range also includes the negative power supply voltage.

The new version uses the TCRT1000 reflective optical sensor for photoplethysmography. The current work is based on the principle of photoplethysmography (PPG) which is a non-invasive method of measuring the variation in blood volume in tissues using a light source and a detector. Since the change in blood volume is synchronous to the heart beat, this technique can be used to calculate the heart rate.

Transmittance and reflectance are two basic types of photoplethysmography. For the transmittance PPG, a light source is emitted in to the tissue and a light detector is placed in the opposite side of the tissue to measure the resultant light. Because of the limited penetration depth of the light through organ tissue, the transmittance PPG is applicable to a restricted body part, such as the finger or the ear lobe. However, in the reflectance PPG, the light source and the light detector are both placed on the same side of a body part. The light is emitted into the tissue and the reflected light is measured by the detector. As the light doesn't have to penetrate the body, the reflectance PPG can be applied to any parts of human body. In either case, the detected light reflected from or transmitted through the body part will fluctuate according to the pulsatile blood flow caused by the beating of the heart.

The figure 2 shows the schematic of heart rate sensor used in the work.

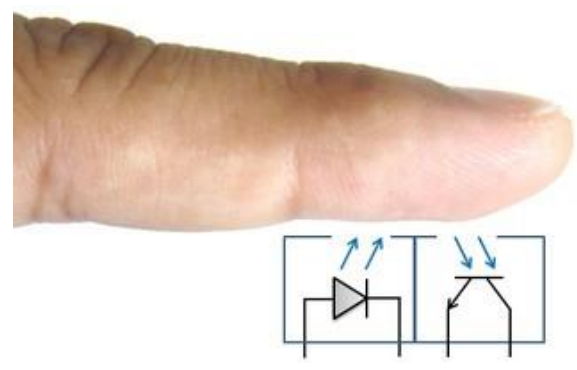

Fig 2. Heart rate sensor TCRT1000

The following picture shows a basic reflectance PPG probe to extract the pulse signal from the fingertip. A subject's finger is illuminated by an infrared light-emitting diode. More or less light is absorbed, depending on the tissue blood volume. Consequently, the reflected light intensity varies with the pulsing of the blood with heart beat. A plot for this variation against time is referred to be a photo plethysmographic or PPG signal.

\section{B. Software description}

The processor used is the Arduino UNO R3 board. The system gets the input from different sensors that measures skin temperature, skin resistance and heartbeat. These raw signals are sensed and processed in the board. The output is in the form of buzzer, LCD and graphical display.

The Arduino Uno is a microcontroller board based on the ATmega328. It has 14 digital input/output pins. 6 analog inputs, a $16 \mathrm{MHz}$ ceramic resonator, a USB connection and a reset button.

After powering the board from a $3-5.5 \mathrm{~V}$ supply, the enable is made high to activate the IR sensor. The finger must be placed still on the sensor. Within a couple seconds the circuit stabilizes and LED flashes synchronously with heart beat. The output voltage waveform can also be viewed on an oscilloscope.

The board receives the raw input signals from all the three sensors and is processed.

\section{RESULTS AND DISCUSSIONS}

The output waveforms from skin resistance sensor, body temperature sensor and heart rate sensors that are processed in the board are shown in the figures 3, 4 and 5 respectively.

The figure 6 shows the circuit of designed lie detector in the current work.
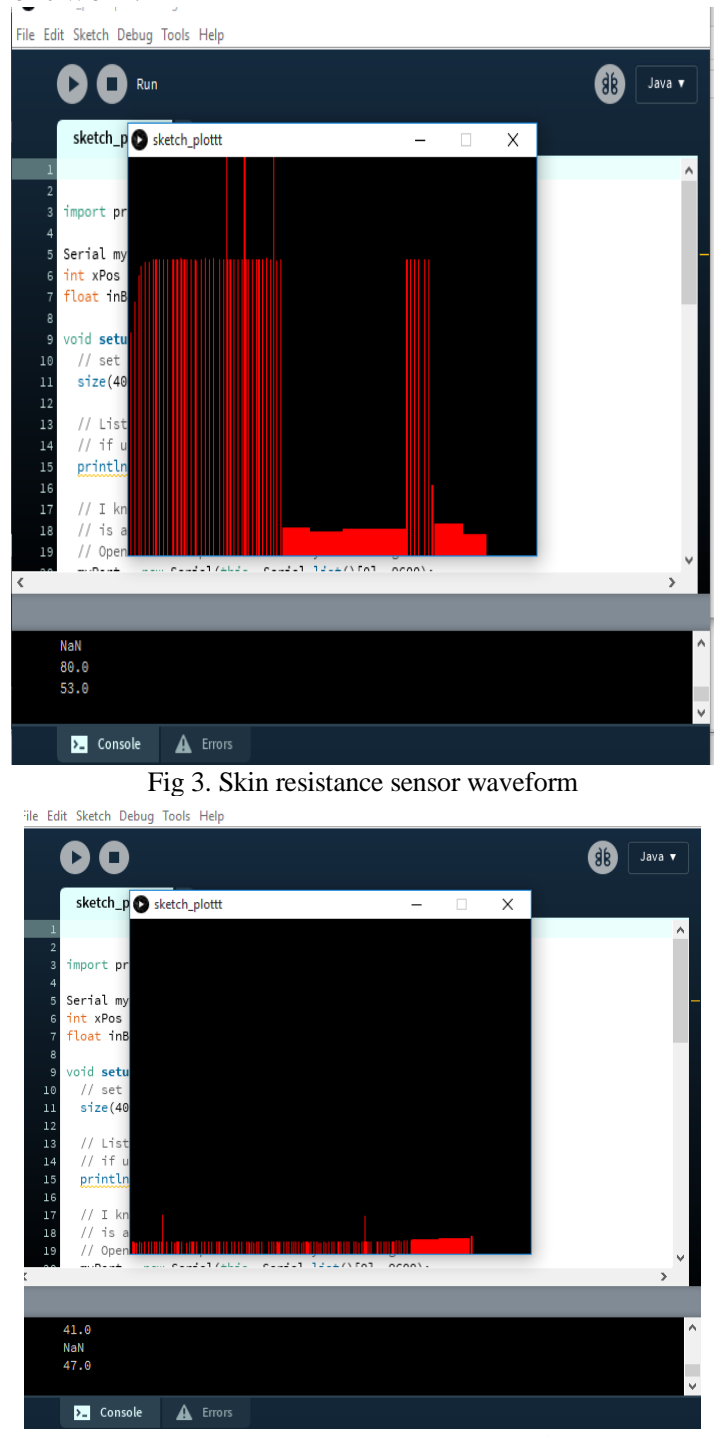

Fig 4. Body temperature sensor waveform 
(3) sketch_plottl Processing 3.0 .2

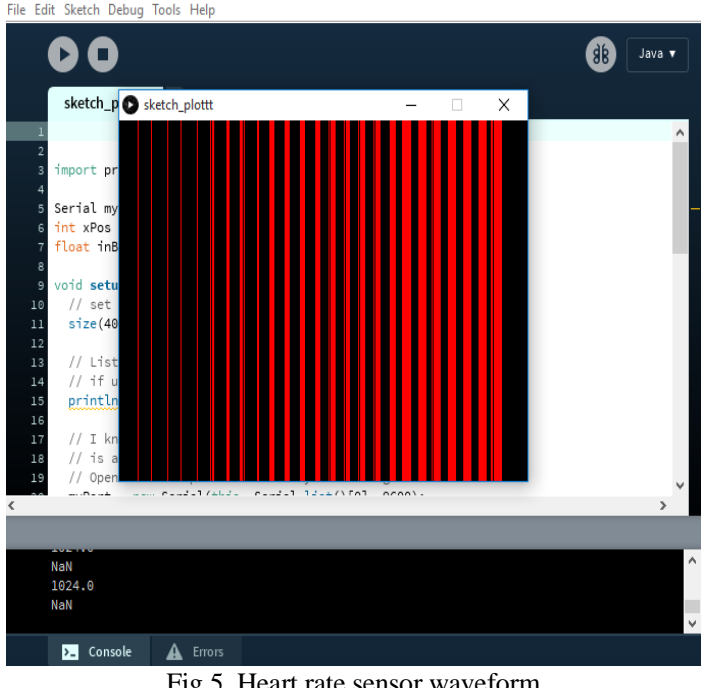

Fig 5. Heart rate sensor waveform

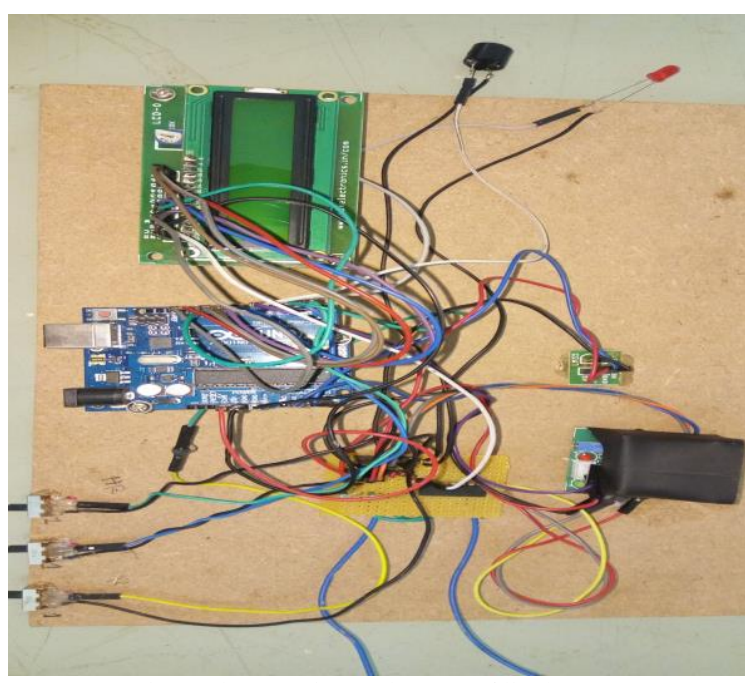

Fig 6. The experimental set up of lie detector

\section{CONCLUSION}

The circuit is mainly designed to determine if subject were actually lying. The physiological parameters like skin resistance, skin temperature and heart rate are found to be the simplest and easily accessible parameters that changes accordingly in response to the questionnaire posed to the subject.

This test is found to be useful to detect theft person by law enforcement. It helps in crime investigation and for counseling a person by psychiatrist.

The proto type designed is found to be simple, portable and cost effective with good accuracy. But if the person subjected to the test is too confident about his responses then the test may not detect as there may not be any sweat or nervous feeling. The test may also fail if the subject is too tensed or mentally weak. In real time, the test can be performed with proper sedations to the subjects that may give more accurate results.

Powerful lie detection tools may someday surpass the accuracy of the polygraph and permanently change how suspects are convicted and freed. A computer analyzes the data and informs the examiner if the suspect's memory holds information about the crime that only the perpetrator could know. The suspect would not even have to speak, for the examiner to know if the subject has exclusive knowledge of the crime. The guilty could be clearly identified and the innocent would be set free.

\section{REFERENCES}

[1] Shamoo AE, "Ethical and regulatory challenges in psychophysiology and neuroscience-based technology for determining behavior". US National Library of Medicine, 17(1): pp. 8-29. DOI: 10.1080/ 08989620903520271, 2010

[2] Seymour TL, Fraynt BR., "Time and encoding effects in the concealed knowledge test", Applied Psychophysiology Biofeedback, US National Library of Medicine, 34(3): pp.177-87. DOI: 10.1007/s10484-009-9092-3. Epub June 1, 2009.

[3] Bunn GC., "Spectacular science: the lie detector's ambivalent powers", Hist Psychology, US National Library of Medicine 10(2): pp.156-78, May 2007

[4] Gannon TA., "Increasing honest responding on cognitive distortions in child molesters: the bogus pipeline procedure", Journal of Interpersonal Violence, doi: 10.1177/0886260505282887, vol. 21 no. 3, pp.358-375, March 2006

[5] Wang Zhiyu, "Based on physiology parameters to design lie detector", 2010 International Conference on Computer Application and System Modeling (ICCASM 2010), vol. 8, pp. 634-637, DOI: $10.1109 / 5619088,2010$ 